\title{
An exact solution approach to warm inflation using Tsallis and Barrow holographic dark energy entropy within Rastall gravity
}

\author{
R. Saleem ${ }^{1,2, a}$, Iqra Shahid ${ }^{1,2, b}$, Mudassar Sabir ${ }^{1,2, c}$ \\ ${ }^{1}$ Department of Mathematics, COMSATS University Islamabad, Lahore Campus, Lahore 54590, Pakistan \\ 2 Institute of Geophysics and Geomatics, China University of Geosciences, Wuhan, Hubei 430074, China
}

Received: 6 October 2021 / Accepted: 15 February 2022

(C) The Author(s), under exclusive licence to Società Italiana di Fisica and Springer-Verlag GmbH Germany, part of Springer Nature 2022

\begin{abstract}
This paper studies the phenomena of warm inflation in a modified cosmological scenario within Rastall gravity. In this context, we modify the standard Friedmann equations using recently proposed Tsallis and Barrow holographic dark energy entropies, alternatively. For both entropies, the exact solutions including the inflaton field, potential required to produce inflation and the scale factor are obtained under slow-roll approximation for low- and high-dissipative regimes. We obtain exponentially growing scale factors for both dark energy models. To confront the theoretical predictions of these models with recent observational data, we calculate the slow-roll parameters, number of e-folds, scalar spectral index, running of scalar spectral index and the tensor-to-scalar ratio for both extremes of dissipation. The Planck bounds on inflationary trajectories are being used to constraint the free parameters. The results are interesting and physically viable up to $2 \sigma$ level.
\end{abstract}

\section{Introduction}

One of the foremost models, which played a vital role in modern cosmology, is the "cosmic inflation" [1]. Numerous analytical predictions of cosmic inflation have been verified by cosmic microwave background radiation (CMBR), Wilkinson Microwave Anisotropy Probe (WMAP) [2] and Planck satellites [3-5]. It seems that all the inflationary models have been explored by considering the cosmological principle [6], which states that our universe is clearly homogeneous and isotropic over large scales as represented by Friedmann Lemaitre-Robertson-Walker (FLRW) spacetime [7]. According to the big-bang cosmology, the universe is in its present state by two possible stages, which could be either radiation-dominated or matter-dominated phases.

The proposition of inflation was first given by Guth [1] and then by Sato [8] independently in 1981. This basic proposition is now termed as "old inflation." It suggests that de Sitter inflation makes use of the first-order transition to true vacuum. However, it appears insufficient when it fails to explain inhomogeneity in the universe, which is created due to bubble collision as soon as the inflation ends. An updated version was proposed by Linde [9], and later by Albrecht and Steinhardt [10] in 1982, which is now termed as "new inflation." It explains the slow-roll inflation with the second-order transition to the true vacuum. However, this proposition has its demerits too as it fails to explain the problem of spending enough time in false vacuum that can result in a significant amount of inflation. Linde [11] studied chaotic inflation, which is known to be a slow-roll inflation with initial chaotic scalar fields. This model proposes that the isotropic and homogeneous universe is produced in regions with sufficient inflation. The models of old and new inflation suggest that universe has been in a state of thermal equilibrium since its beginning, and chaotic inflation believes that such thermal equilibrium was not a necessity. Furthermore, chaotic inflation coincides with Planck density, thus solving the issues of initial conditions.

Inflation can be classified into warm inflation (WI) and supercooled inflation. In supercooled type of inflation, the dissipative effects introduced by a term $\Gamma$ are absent, so no particles are assembled and the total energy of the system remains conserved in classical inflaton field that is slowly rolling. On the contrary, WI [12] is a procedure in which radiation production takes place during inflationary era, which generates particles at relativistic scale. It combines the end phase of inflation with recent cosmic phase. In the models with WI, radiation production is preserved by $\Gamma$, which is added separately in the conservation equation of the homogeneous scalar field. It can be assumed as a constant term, function of temperature, function of inflaton or a combination of both. According to second law of thermodynamics, $\Gamma$ should be positive, which results in exchange of inflaton's density to radiation density. The significance of WI is generally measured by $r_{*}=\frac{\Gamma}{3 H}$. The factor of $\Gamma$ in contrast to $H$ leads to two extremes in WI, which are strong dissipation $\left(r_{*} \gg 1\right)$ and weak dissipation $\left(r_{*} \ll 1\right)$.

\footnotetext{
a e-mail: rabiasaleem@cuilahore.edu.pk (corresponding author)

b e-mail: ikra.2016@gmail.com

c e-mail: sabir@cug.edu.cn
} 
In general relativity (GR), the total mass (rest energy) of a system remains preserved. However, to support this statement there is no experimental evidence. Consequently, there are some terms for GR, which do not care about this covariant divergence of energy momentum tensor (EMT). Rastall $[13,14]$ comes up with modified theory of gravity in 1972 with non-conserved EMT and lately discussed extensively in literature. Analytically, the matter source is expressed by the EMT as in GR and further by the metric of the external space. There are two major demerits of this modified theory: First one is its phenomenological nature, and second is that there is no action from which Rastall theory can be obtained. However, with fundamental aspects of gravity theory, its rich structure can be associated. Furthermore, as there is no possibility for an action which could be implied to derive Rastall theory, a chance occurs for an action when an external field is set up in the Einstein-Hilbert action with some Lagrange multiplier. Also, geometrical frameworks like Weyl geometry can derive related field equations as Rastall theory [15,16]. Rastall gravity (RG) has been used widely in different cosmological scenarios [17-19]. The particle creation process [20-23] and this modified theory are relatable in cosmic evolution, while both of them do not hold the conservation of the EMT.

From the latest observational data, the dark region of our universe is filled out with two fluids, known to be dark matter (DM) and dark energy (DE) [24,25]. Dark matter is responsible for giving shape to the universe, while DE manages the present accelerating phase of the universe. By the combination of DM and DE, total $96 \%$ of energy density is being obtained, where the amount of DE and DM in total energy density budget is about $68 \%$ and $28 \%$, respectively. As different types of cosmological models have been analyzed and proposed in past years. The simplest model of the universe is non-interacting model in which DM and DE conserved individually, while an interaction between DM and DE is also considered in the literature [26-30].

In recent years, specific attention has been attracted to nonadditive systems such as gravitational system, and the general Boltzmann-Gibbs (BG) additive entropy converts into non-extensive Tsallis entropy [31-33]. Tsallis entropy increases the interest of investigating a viable extension of Shannon's entropy to statistical theory. This curiosity raised mainly on account of similarities between BG and Shannon entropy functions. With different motivations, all models lead to some conversion for area law entropy. Like, loop quantum gravity as a result of quantum fluctuations and thermal equilibrium fluctuations [34-37] or else the entropies of cosmological models that combine the late-time acceleration and inflation [38] give logarithmic corrections. However, the complexity of quantum fields in the interior and exterior of the horizon $[39,40]$ brings out some modification in area law of entropy. In gravitational system with some variation in partition function, where the BG theory cannot be tested, one has to adopt the nonadditive entropy (Tsallis entropy). Thus, the mathematical form of thermodynamical entropy of a black hole (BH) can be expressed as

$$
S=\alpha A^{b}
$$

where $\alpha$ is an unknown constant, $A$ is the $\mathrm{BH}$ horizon area, and $b$ is Tsallis parameter. In cosmology, the establishment of Tsallis entropy gives modification of Friedmann equations that hold extra terms and convert it into standard cosmological model when the generalized Tsallis entropy becomes conventional.

Recently, many researchers have again revisited holographic DE (HDE) models and analyzed it in various contexts [41-44]. The authors in Refs. [45,46] established the "Barrow HDE" (BHDE). They applied the holographic principle on cosmological framework and Barrow entropy comparatively the familiar "Bekenstein-Hawking entropy" (BHE). The cosmological consequences of BHDE and its thermodynamic analysis by incorporating viscosity are presented in [47]. They presented a reconstruction scheme for the parameters associated with BHDE under the purview of viscous cosmology. The authors in [48] discussed BHDE for the non-flat universe. They derived the differential equations that govern the evolution of the DE density parameter and presented an analytical methodology for the associated DE EoS parameter. The BHDE scenario is constrained by the authors in a paper [49] using observational data coming from the Supernovae (SNIa) Pantheon sample along with precise observations of the Hubble parameter from cosmic chronometers sample. In a recent paper [50], an interacting BHDE model has been proposed using Barrow entropy. They investigated the evolution of a spatially flat FLRW universe made up of pressure-less DM and BHDE that interacted via a well-motivated interaction term. In [51], authors reconstruct $f(R)$ gravity using BHDE as the type of background evolution and discovered that the EoS parameter can transit from quintessence to phantom. The conditions on the power-law variable $\alpha$ are obtained in the quintessence- and phantom-dominated regimes and also set some restrictions under which generalized second law of thermodynamics fails in a cosmological scenario [52]. The Tsallis and Rényi entropies were considered as possible alternatives to the BHDE in [53]. In [54], authors proposed a new entropy that generalizes the Rényi, Tsallis, Sharma-Mittal, Kaniadakis, Barrow and loop quantum gravity entropies and is reduced to the Barrow holographic entropy under a certain limit. Reconstruction schemes for tachyon, k-essence and dilaton scalar field DE models through Tsallis holographic dark fluid in the framework of Chern-Simons modified gravity are presented in [55]. In [56], author has established the exact solution approach for WI in a Tsallis entropy-modified Friedmann universe. They showed that their method generalizes the modified Friedmann approach of Del Campo and reduces to the well-known Hamilton-Jacobi formalism for inflation in specific limits.

The paper is being structured as follows: In Sect. 2, the basic formalism to discuss WI within RG is given. Exact solutions by employing the well-known slow-roll approximation are found. The general perturbation analysis is performed in Sect. 3 during highand low-dissipative regimes. In Sects. 4 and 5, the Friedmann universe is modified using Tsallis and BHDE entropy, respectively. In modified universe, the exact solutions as well as the inflationary observable are being developed under both entropies and observed graphically. The work is summarized in the last section. 


\section{Inflationary formalism within Rastall gravity under slow-roll approximation}

The general Einstein field equations for RG can be written as

$$
G_{\mu \nu}=\kappa S_{\mu \nu},
$$

where $G_{\mu \nu}=R_{\mu \nu}-\frac{1}{2} R g_{\mu \nu}$ is the Einstein tensor with Ricci tensor $R_{\mu \nu}$, Ricci scalar $R$, metric tensor $g_{\mu \nu}$ and an additional parameter $\bar{\lambda}$. The effective energy-momentum tensor is defined as

$$
\begin{aligned}
\mathcal{S}_{\mu \nu} & =T_{\mu \nu}-\frac{\kappa \bar{\lambda}}{4 \kappa \bar{\lambda}-1} g_{\mu \nu} T, \\
T_{\mu \nu} & =(\bar{\rho}+\bar{p}) u_{\mu} u_{\nu}-\bar{p} g_{\mu \nu} .
\end{aligned}
$$

The corresponding FRW equations for flat spacetime can be obtained as follows

$$
\begin{aligned}
3(1-4 \kappa \bar{\lambda}) H^{2}-6 \kappa \bar{\lambda} \dot{H} & =\kappa \bar{\rho}, \\
3(1-4 \kappa \bar{\lambda}) H^{2}+2(1-3 \kappa \bar{\lambda}) \dot{H} & =-\kappa \bar{P},
\end{aligned}
$$

where derivative with respect to time is represented by a dot. For $\bar{\lambda}=0$, the equations return back to flat FRW in GR.

To solve the system of equations analytically, an equation of state $\bar{P}_{\gamma}=(\gamma-1) \bar{\rho}_{\gamma}$, where $\frac{2}{3} \leq \gamma \leq 2$ is being used as a constraint. Combining this constraint with field equations, we get following expression of $\dot{H}$, independent of Rastall parameter

$$
\dot{H}=-\frac{\kappa}{2}\left(\gamma \bar{\rho}_{\gamma}\right)
$$

Substituting the above expression back into first field equation, we get Hubble expansion

$$
H^{2}=\left(\frac{\kappa(3 \kappa \bar{\lambda} \gamma-1)}{4 \kappa \bar{\lambda}-1}\right)\left(\bar{\rho}_{\gamma}-\frac{\bar{\rho}_{B}}{3 \kappa \bar{\lambda} \gamma-1}\right)
$$

where the term $\bar{\rho}$ includes both (inflaton $\bar{\rho}_{B}$ and radiation $\bar{\rho}_{\gamma}$ ) energy densities. Since it is considered that $\bar{\rho}_{B} \gg \bar{\rho}_{\gamma}$ during inflation, therefore, Eq. (6) is reduced to

$$
H^{2}=\frac{\kappa}{1-4 \kappa \bar{\lambda}} \bar{\rho}_{B}
$$

where scalar field energy density has standard form

$$
\bar{\rho}_{B}=\frac{3}{2}\left(\dot{B}^{2}+V(B)\right) .
$$

The following equation of continuity can be obtained using second Bianchi identity

$$
G_{v ; \mu}^{\mu}=0 \Rightarrow\left(\frac{3 \kappa \bar{\lambda}-1}{4 \kappa \bar{\lambda}-1}\right) \dot{\bar{\rho}}+\left(\frac{3 \kappa \bar{\lambda}}{4 \kappa \bar{\lambda}-1}\right) \dot{\bar{P}}+3 H(\bar{\rho}+\bar{P})=0 .
$$

The dynamics of WI in spatially flat FRW universe is presented by the following equations

$$
\begin{aligned}
\left(\frac{1-3 \kappa \bar{\lambda} \gamma}{1-4 \kappa \bar{\lambda}}\right) \dot{\bar{\rho}}_{B}+3 H\left(\bar{\rho}_{B}+\bar{P}_{B}\right) & =-\Gamma \dot{B}^{2}, \\
\left(\frac{1-3 \kappa \bar{\lambda}}{1-4 \kappa \bar{\lambda}}\right) \dot{\bar{\rho}}_{\gamma}+4 H \bar{\rho}_{\gamma} & =\Gamma \dot{B}^{2},
\end{aligned}
$$

where $\Gamma$ is a dissipation coefficient of inflaton. By defining a term $r_{*}=\frac{\Gamma}{3 H}$, Eq. (11) can be written as

$$
\left(\frac{1-3 \kappa \bar{\lambda}}{1-4 \kappa \bar{\lambda}}\right) \dot{\bar{\rho}}_{\gamma}+4 H \bar{\rho}_{\gamma}=3 H r_{*} \dot{B}^{2}
$$

Let us consider $\dot{\bar{\rho}}_{\gamma} \approx 0$ during inflation, above equation leads us to the solution of radiation density

$$
\bar{\rho}_{\gamma}=\frac{3}{4} r_{*} \dot{B}^{2} .
$$

To make the system easy to handle, slow-roll conditions are implemented given as under

$$
\dot{\bar{\rho}}_{\gamma} \leq 4 H \bar{\rho}_{\gamma}, \quad \dot{\bar{\rho}}_{\gamma} \ll \Gamma \dot{B}^{2}, \quad \bar{\rho}_{B} \sim V, \quad \dot{B}^{2} \ll V(B), \quad \ddot{B} \ll\left(3 H+\frac{\Gamma}{3}\right) \dot{B} .
$$


Under above-mentioned approximations, the inflaton's potential comes out to be

$$
V^{\prime}(B)=-9 H \dot{B}\left(\frac{1-4 \kappa \bar{\lambda}}{1-3 \kappa \bar{\lambda} \gamma}\right)\left(1+\frac{r_{*}}{3}\right) .
$$

The general from of the Friedmann equation can be written as

$$
F(H)=-\frac{3 \kappa}{2(4 \kappa \bar{\lambda}-1)}\left(\dot{B}^{2}+V(B)\right)=\frac{\kappa}{4 \kappa \bar{\lambda}-1} \bar{\rho}_{B} .
$$

Under the condition $\dot{B}^{2} \ll V(B)$, the above equation is further reduced to

$$
F(H)=-\frac{3 \kappa V(B)}{2(4 \kappa \bar{\lambda}-1)} ;
$$

taking derivative of $F(H)$ with respect to field, we get

$$
F_{, H} H^{\prime}(B)=-\frac{3 \kappa}{2(4 \kappa \bar{\lambda}-1)} V^{\prime}(B) .
$$

By putting the value of $V^{\prime}(B)$ from Eq. (13) in the above equation, we get the expression of $\dot{B}$ as

$$
\dot{B}=-\frac{2(1-3 \kappa \bar{\lambda} \gamma)}{9 \kappa}\left(\frac{F_{, H} H^{\prime}}{H\left(3+r_{*}\right)}\right) .
$$

After some algebra, we can find $\bar{\rho}_{\gamma}$ making use of Eq. (12)

$$
\bar{\rho}_{\gamma}=\frac{3 r_{*}}{4}\left(\frac{2(1-3 \kappa \bar{\lambda} \gamma)}{9 \kappa}\left(\frac{F_{, H} H^{\prime}}{H\left(3+r_{*}\right)}\right)\right)^{2} .
$$

Now, we can easily develop a relationship between $\bar{\rho}_{\gamma}$ and $\bar{\rho}_{B}$ as follows

$$
\bar{\rho}_{\gamma}=\frac{r_{*}}{27 \kappa F} \frac{(1-3 \kappa \bar{\lambda} \gamma)^{2}}{(4 \kappa \bar{\lambda}-1)}\left(\frac{F_{, H} H^{\prime}}{H\left(3+r_{*}\right)}\right)^{2} \bar{\rho}_{B} .
$$

As $\bar{\rho}_{\gamma}$ is the pure radiation, we can write $\bar{\rho}_{\gamma}=\sigma T^{4}$, where $\sigma$ is the "Stefan-Boltzmann constant" and $T$ is the temperature of radiation bath. Combining Eq. (18) with above relation of $\bar{\rho}_{\gamma}$, we get exact solution of the temperature as

$$
T=\left(\frac{3 r_{*}}{4 \alpha}\right)^{\frac{1}{4}}\left(\frac{2(1-3 \kappa \bar{\lambda} \gamma)}{9 \kappa}\left(\frac{F_{, H} H^{\prime}}{H\left(3+r_{*}\right)}\right)\right)^{\frac{1}{2}} .
$$

Using Eq. (17) in Eq. (14), effective potential can be written as

$$
\begin{aligned}
V(B) & =-\frac{2(4 \kappa \bar{\lambda}-1) F}{3 \kappa}-\frac{2(1-3 \kappa \bar{\lambda} \gamma)}{9 \kappa} \frac{F_{, H} H^{\prime}}{H\left(3+r_{*}\right)}, \\
& =\left(-\frac{2}{3}-\frac{4(1-3 \kappa \bar{\lambda} \gamma)^{2}}{81 \kappa F(4 \kappa \bar{\lambda}-1)}\left(\frac{F_{, H} H^{\prime}}{H\left(3+r_{*}\right)}\right)^{2}\right) \bar{\rho}_{B} .
\end{aligned}
$$

The amount of inflation is determined by e-folding number, given as

$$
N=\int_{t}^{t_{*}} H(t) d t=-\frac{9 \kappa}{2(1-3 \kappa \bar{\lambda} \gamma)} \int_{B_{e}}^{B_{*}}\left(\frac{H^{2}\left(3+r_{*}\right)}{F_{, H} H^{\prime}}\right) d B .
$$

By definition, we have $d N=\frac{d a}{a}$, yielding following solution of the scale factor

$$
a\left(B_{e}\right)=a\left(B_{*}\right) \exp \left[\frac{9 \kappa}{2(1-3 \kappa \bar{\lambda} \gamma)} \int_{B_{e}}^{B_{*}}\left(\frac{H^{2}\left(3+r_{*}\right)}{F_{, H} H^{\prime}}\right) d B\right] .
$$

The slow-roll parameters $\epsilon, \eta$ are developed for considered model via following definitions

$$
\begin{aligned}
& \epsilon=-\frac{\dot{H}}{H^{2}}=\frac{2(1-3 \kappa \bar{\lambda} \gamma)}{9 \kappa}\left(\frac{F_{, H}}{H\left(3+r_{*}\right)}\left(\frac{H^{\prime}}{H}\right)^{2}\right), \\
& \eta=-\frac{\ddot{H}}{2 H \dot{H}}=\frac{(1-3 \kappa \bar{\lambda} \gamma)}{9 \kappa}\left(\frac{F_{, H}}{H\left(3+r_{*}\right)}\left(\frac{H^{\prime \prime}}{H}\right)\right) .
\end{aligned}
$$


One of the important and foremost slow-roll conditions is

$$
-\frac{\ddot{H}}{\dot{H} H} \ll 1
$$

Now, our target is to evaluate this term $-\frac{\ddot{H}}{\dot{H} H}$, to find out some new parameters. We start by observing second derivative of $H$, i.e., $\ddot{H}=\frac{d}{d t}\left(H^{\prime} \dot{B}\right)$. By expanding derivative in the above equation, we get

$$
\ddot{H}=\dot{B}^{2} H^{\prime \prime}+\ddot{B} H^{\prime} .
$$

To evaluate Eq. (24), we first find out the value of $\ddot{B}$. We take derivative of Eq. (17) with respect to $t$, which leads us to

$$
\ddot{B}=-\frac{2(1-3 \kappa \bar{\lambda} \gamma)}{3 \kappa}\left(\frac{(9 H+\Gamma)\left(H^{\prime 2} F_{\prime} H H+F^{\prime} H H^{\prime \prime}\right)-F_{\prime} H H^{\prime}\left(H^{\prime}+\Gamma^{\prime}\right)}{(9 H+\Gamma)^{2}}\right) \dot{B},
$$

which enables us to achieve

$$
-\frac{\ddot{H}}{\dot{H} H}=4 \eta+\frac{2(1-3 \kappa \bar{\lambda} \gamma)}{9 \kappa}\left(\frac{H^{\prime 2} F_{\prime} H H}{H^{2}\left(3+r_{*}\right)}-18\left(\frac{r_{*}}{1+r_{*}}\right) \frac{H^{\prime 2} F_{\prime} H}{\Gamma H^{2}\left(3+r_{*}\right)}+\frac{H^{\prime} \Gamma^{\prime} F_{\prime} H}{\Gamma H^{2}\left(3+r_{*}\right)}\right) .
$$

The above equation allows us to rewrite it as

$$
-\frac{\ddot{H}}{\dot{H} H}=4 \eta+\chi-\frac{r_{*}}{1+r_{*}}(9 \xi+\beta),
$$

where

$$
\begin{aligned}
& \chi=\frac{2(1-3 \kappa \bar{\lambda} \gamma)}{9 \kappa H^{2}} \frac{H^{\prime 2} F^{\prime} H H}{3+r_{*}}, \\
& \xi=\frac{2(1-3 \kappa \bar{\lambda} \gamma)}{\kappa H^{2}} \frac{H^{\prime 2} F_{\prime} H}{\Gamma\left(3+r_{*}\right)}, \\
& \beta=\frac{2(1-3 \kappa \bar{\lambda} \gamma)}{9 \kappa H^{2}} \frac{H^{\prime} \Gamma^{\prime} F_{\prime} H}{\Gamma\left(3+r_{*}\right)} .
\end{aligned}
$$

For perturbation analysis, we defined some other parameters above that will be useful in further calculations.

\section{Perturbation spectra analysis}

The amplitude of adiabatic perturbations is given by [57]

$$
P_{s}(k)=\frac{4}{25}\left(\frac{H}{|\dot{B}|}\right)^{2} d B^{2}
$$

where $\dot{B}$ is given in Eq. (17).

3.1 For high-dissipative regime $\left(r_{*} \gg 1\right)$

During high dissipation, the term $d B^{2}$ is defined by

$$
d B^{2}=\frac{k_{F} T}{2 \pi}
$$

with $k_{F}=\sqrt{\Gamma H}$ being the "freeze out number." Now using Eq. (20) and expression of $\epsilon$ given in Eq. (23), we can develop the result for $P_{S}(k)$ for our model as under

$$
P_{S}(k)=\frac{2}{25 \pi}\left(\frac{3 r_{*}}{4 \alpha}\right)^{\frac{1}{4}} \sqrt{\Gamma H}\left(\frac{\epsilon H^{2}}{H^{\prime}}\right)^{\frac{1}{2}}\left(\frac{H^{\prime}}{\epsilon H}\right)^{2} .
$$

In this regime, the scalar spectral index $n_{s}$ and its running $\alpha_{s}$ have the following forms

$$
\begin{aligned}
& n_{s}=1+\frac{d \ln P_{s}(k)}{d \ln k}=1-3 \eta+\frac{3}{2} \chi+\frac{9}{4} \epsilon+\frac{3}{4} \beta+\frac{r_{*}}{3+r_{*}}\left(\frac{27}{2} \xi+\frac{3}{2} \beta\right), \\
& \alpha_{s}=\frac{d n_{s}}{d \ln k}=-\frac{3}{2} \epsilon v-6 \eta \epsilon-15 \chi \epsilon+\frac{3}{2} \epsilon \psi+\frac{9}{2} \epsilon^{2}+\frac{3}{4} \delta-\frac{3}{4} \beta^{2}+\frac{3}{2} \eta \beta+\frac{3}{4} \chi \beta
\end{aligned}
$$




$$
\begin{aligned}
& +\frac{r_{*}}{3+r_{*}}\left(36 \eta \xi-15 \chi \xi-3 \chi \beta+\frac{135}{4} \xi \epsilon+\frac{15}{4} \beta \epsilon-\frac{3}{4} \beta^{2}-\frac{27}{4} \xi \beta\right. \\
& \left.+\frac{3}{2} \delta+\frac{27}{4} \beta \xi+\frac{3}{2} \delta\right)+\frac{r_{*}^{2}}{\left(3+r_{*}\right)^{2}}\left(\frac{261}{2} \xi^{2}+\frac{83}{2} \beta \xi+3 \beta^{2}\right),
\end{aligned}
$$

where

$$
\begin{aligned}
& \delta=\left(\frac{2(1-3 \kappa \bar{\lambda} \gamma)}{9 \kappa}\right)^{2}\left(\frac{F_{\prime_{H}}}{H\left(3+r_{*}\right)}\right)^{2} \frac{H^{\prime 2} \Gamma^{\prime \prime}}{H \Gamma}, \\
& v=\left(\frac{2(1-3 \kappa \bar{\lambda} \gamma)}{9 \kappa}\right)\left(\frac{F_{\prime} H}{H\left(3+r_{*}\right)}\right) \frac{H^{\prime \prime \prime}}{H^{\prime}}, \\
& \psi=\left(\frac{2(1-3 \kappa \bar{\lambda} \gamma)}{9 \kappa}\right)\left(\frac{F_{\prime} H H H H^{\prime 2}}{H\left(3+r_{*}\right)}\right) .
\end{aligned}
$$

The tensor power spectrum is defined as [58]

$$
P_{T}(k)=\frac{32}{75 m_{p}^{4}} V(B)
$$

where $V \approx \frac{2(1-4 \kappa \bar{\lambda}) F}{3 \kappa}$ during inflation. Tensor-to-scalar ratio is calculated as

$$
r=\frac{P_{T}(k)}{P_{S}(k)}=\frac{64(1-4 \kappa \bar{\lambda}) F}{225 m_{p}^{4} \kappa}\left(\left(\frac{16}{1562500 \alpha \pi^{4}}\right)^{\frac{1}{2}} \Gamma^{\frac{3}{2}} H^{-\frac{3}{2}} \epsilon^{-3} H^{\prime 3}\right)^{-\frac{1}{2}} .
$$

3.2 For low-dissipative regime $\left(r_{*} \ll 1\right)$

The amplitude of the scalar spectrum is expressed by the same Eq. (26), but the term $d B$ is defined differently as $d B=H T$. Power spectrum $P_{S}(k)$ for the low-dissipative regime turns out to be

$$
P_{S}(k)=\frac{4}{25}\left(\frac{T^{2} H^{\prime 2}}{\epsilon^{2}}\right),
$$

which is equivalent to

$$
P_{s}(k)=\left(\left(\frac{4}{625 \alpha}\right) \epsilon^{-2} H^{\prime 2} \Gamma H^{3}\right)^{\frac{1}{2}} .
$$

In a similar pattern as we do in high-dissipation regime, we evaluate $n_{s}, \alpha_{s}$ for low dissipation as

$$
\begin{aligned}
n_{s}= & 1+\frac{7}{2} \epsilon-\frac{1}{2} \beta-3 \eta-\chi+\frac{r_{*}}{3+r_{*}}(9 \xi+\beta), \\
\alpha_{s}= & -11 \epsilon \eta-\frac{5}{2} \chi \epsilon-\frac{1}{2} \delta+\frac{1}{2} \beta^{2}-2 \eta \beta+\epsilon \beta-\chi \beta-\frac{3}{2} \epsilon v-5 \eta \chi-\epsilon \psi \\
& +\frac{r_{*}}{3+r_{*}}(-9 \epsilon \xi-\epsilon \beta+9 \eta \xi+\eta \beta+\chi \xi+\chi \beta)+\frac{r_{*}^{2}}{\left(3+r_{*}\right)^{2}}\left(81 \xi^{2}+\beta^{2}+18 \beta \xi\right) .
\end{aligned}
$$

The parameter $r$ for low-dissipative regime can be obtained as

$$
r=\frac{P_{T}(k)}{P_{s}(k)}=\frac{32(1-4 \kappa \bar{\lambda}) F}{9 m_{p}^{4} \kappa}\left(\Gamma H^{\prime 2} \epsilon^{-2} H^{3}\right)^{-\frac{1}{2}} .
$$

Now, we have done all the theoretical basis for our approach. Next, we will apply it on a modified universe models with Tsallis entropy and Barrow HDE as well.

\section{Warm inflation using Tsallis entropy within Rastall gravity}

The modified Friedmann universe with Tsallis entropy has the following form of corresponding equation

$$
F(H)=H^{2(2-k)} .
$$


In our approach, we will use both $H$ and $\Gamma$ as a function of inflaton, $B$. We consider $H$ and $\Gamma$ to be the power-law functions of inflaton, defined as

$$
H(B)=H_{0} B^{n}, \quad \Gamma(B)=\Gamma_{0} B^{m},
$$

where $H_{0}, \Gamma_{0}$ are the arbitrary constants, and the exponent $n$ and $m$ are left undetermined here, and we will use the Planck data to observe which power laws hold for underlying model.

By setting $\epsilon=1$ (inflation terminates), we get the value of inflaton field at the time of horizon exit as

$$
B_{e}^{m+2-n(3-2 k)}=\frac{4(1-3 \kappa \bar{\lambda} \gamma)(2-k) n^{2} H^{3-2 k}}{3 \kappa \Gamma_{0}} .
$$

To find the exact solution of the field $B$, we concluded it by the aid of number of e-foldings, given in the following equation

$$
N=-\int_{B_{*}}^{B_{e}} \frac{3 k}{4(1-3 k \bar{\lambda} \gamma)(2-k)}\left(\frac{\Gamma_{0} B^{n} B^{m}}{H_{0}^{3-2 k} B^{n(3-2 k)} B^{n-1}}\right) d B,
$$

yielding

$$
B_{*}=B_{e}\left(1+\frac{(m+2-n(3-2 k)) N}{n}\right)^{\frac{1}{m+2-n(3-2 k)}}
$$

By using Eq. (17), we can write

$$
\dot{B}=\frac{4 n(3 k \bar{\lambda} r-1)(2-k) H_{0}^{4-2 k} B^{n(3-2 k)} B^{n-1}}{3 \kappa \Gamma_{0} B_{*}^{m}}
$$

on integrating from $t_{0}$ to some time $t$, we get

$$
\begin{aligned}
B_{*}(t)^{m+2-n(4-2 k)}= & \left(\frac{n(4-2 k)-2-m}{n}\right) B_{e}^{m+2-n(3-2 k)} H_{0}\left(t-t_{0}\right) \\
& +\left(B_{e}^{m+2-n(3-2 k)}\left(1+\frac{m+2-n(3-2 k) N}{n}\right)\right)^{\frac{m+2-n(4-2 k)}{m+2-n(3-2 k)}} .
\end{aligned}
$$

This allows us to calculate $H(t)$ and $\Gamma(t)$ in the following form

$$
\begin{aligned}
H(t)= & H_{0}\left(\left(\frac{n(4-2 k)-2-m}{n}\right) B_{e}^{m+2-n(3-2 k)} H_{0}\left(t-t_{0}\right)\right. \\
& \left.+\left(B_{e}^{m+2-n(3-2 k)}\left(1+\frac{m+2-n(3-2 k) N}{n}\right)\right)^{\frac{m+2-n(4-2 k)}{m+2-n(3-2 k)}}\right)^{\frac{n}{m+2-n(4-2 k)}}, \\
\Gamma(t)= & \Gamma_{0}\left(\left(\frac{n(4-2 k)-2-m}{n}\right) B_{e}^{m+2-n(3-2 k)} H_{0}\left(t-t_{0}\right)\right. \\
& \left.+\left(B_{e}^{m+2-n(3-2 k)}\left(1+\frac{m+2-n(3-2 k) N}{n}\right)\right)^{\frac{m+2-n(4-2 k)}{m+2-n(3-2 k)}}\right)^{\frac{m}{m+2-n(4-2 k)}} .
\end{aligned}
$$

The potential required to produce inflation is given as

$$
V(B)=\frac{2(1-4 \kappa \bar{\lambda}) F}{3 \kappa}-\left(\frac{4 n(1-3 \kappa \bar{\lambda} \gamma)(2-k) H_{0}^{4-2 k} B^{n(4-2 k)-1}}{3 \kappa \Gamma_{0} B_{*}^{m}}\right)^{2} .
$$

The scale factor as a time function for Tsallis entropy can be found as

$$
a(t)=a_{0} \exp \left(\frac{3 \kappa \Gamma_{0} B_{e}^{m-n(3-2 k)+2}(n-(m-n(3-2 k)+2) N)}{4 n(1-3 \kappa \bar{\lambda} \gamma)(2-k) H_{0}^{3-2 k}(m-n(3-2 k)+2)}\right) .
$$

The scale factor evaluated in Eq. (36) is illustrated graphically in Fig. 1 for different values of $m, n, \bar{\lambda}$. It exhibits positive exponentially increasing behavior as required during inflation.

Detailed analysis of WI in high-dissipation regime in such cosmology can be carried out by having suitable values of the free parameters $\kappa, \bar{\lambda}, m$ and $n$, which are in accordance with the observational data [59]. For this purpose, some significant observational quantitative data are collected, which includes scalar spectral index and its running at the time of horizon exit. The task can be 
Fig. 1 Plot of $a(t)$ for $\bar{\lambda}=1,1.5$

Here, we fixed

$H_{0}=k=1=\kappa=1, \Gamma_{0}=0.5$,

$\gamma=0.16$ and $t_{0}=0.2$. The

parameters $m, n$ are chosen to be

$5,6,7$, and the number of e-folds

is fixed to $N=60$

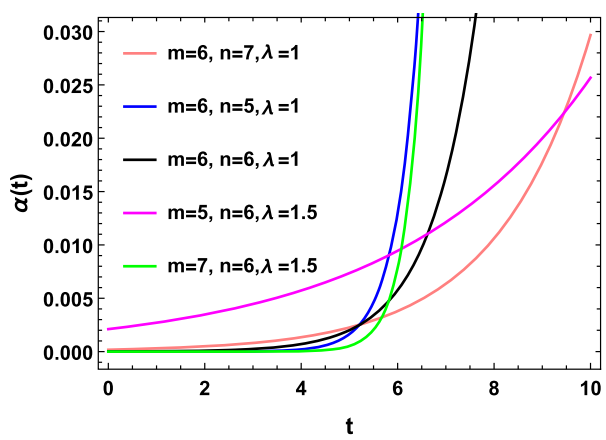

completed by an evaluation of the slow-roll and other parameters as explained earlier at the time of horizon exit. Using Eq. (35), slow-roll parameters can be evaluated at the time of horizon exit. In particular, $\epsilon$ is as under

$$
\epsilon=\frac{1}{H_{0}}\left(\frac{n}{B(m-n(3-2 k)+2)+n}\right)^{\frac{m-n(2-2 k)+2}{n}} \times\left(\frac{3 \Gamma_{0} \kappa}{4(2-k) n^{2} H_{0}^{3-2 k}(1-3 \kappa \bar{\lambda} \gamma)}\right)^{\frac{m-n(3-2 k)+2}{n}} .
$$

The significance of the above-mentioned parameter lies in the fact that it can be used to evaluate some other parameters, which are mentioned in the previous section. Expression of all such parameters at the horizon exit is:

$$
\begin{aligned}
& \beta=\frac{m}{9 n} \epsilon, \quad \xi=\frac{H_{0}}{9 \Gamma_{0}} \epsilon, \quad v=\frac{(n-1)(n-2)}{9 n^{2}} \epsilon, \quad \delta=\frac{m(m-1)}{n} \epsilon^{2}, \\
& \psi=\frac{(2-2 k)(3-2 k)}{n} \epsilon, \quad \eta=\frac{(n-1)}{18 n} \epsilon, \quad \chi=\frac{(3-2 k)}{9} \epsilon .
\end{aligned}
$$

Using these definitions, we reached at the following expressions for low-dissipative regime

$$
\begin{aligned}
n_{s}= & 1+\left(\frac{7}{2}-\frac{n-1}{6 n}-\frac{3-2 k}{9}-\frac{m}{18 n}+\frac{1}{3}\left(\frac{H_{0}}{\Gamma_{0}}+\frac{m}{9 n}\right)\right) \epsilon, \\
\alpha_{s}= & \left(-\frac{11(n-1)}{18 n}-\frac{5(3-2 k)}{18}-\frac{m(m-1)}{2 n}+\frac{m^{2}}{162 n^{2}}-\frac{m(n-1)}{81 n}+\frac{m}{9 n}\right. \\
& -\frac{m(3-2 k)}{81 n}-\frac{(n-1)(n-2)}{6 n^{2}}-\frac{5(n-1)(3-2 k)}{162 n}-\frac{(2-2 k)(3-2 k)}{n} \\
& +\frac{1}{3}\left(-\frac{H_{0}}{\Gamma_{0}}-\frac{m}{9 n}+\frac{H_{0}(n-1)}{18 \Gamma_{0} n}-\frac{m(n-1)}{162 n^{2}}+\frac{H_{0}(3-2 k)}{81 \Gamma_{0}}\right. \\
& \left.\left.+\frac{m(3-2 k)}{81 n}\right)+\frac{1}{9}\left(\frac{H_{0}^{2}}{\Gamma_{0}^{2}}+\frac{m H_{0}}{n \Gamma_{0}}+\frac{m^{2}}{81 n^{2}}\right)\right) \epsilon^{2},
\end{aligned}
$$

and tensor-to-scalar ratio for low-dissipative regime is given by

$$
\begin{aligned}
r= & \frac{32(1-4 \kappa \bar{\lambda}) H_{0}^{\frac{3}{2}-2 k} \epsilon}{9 m_{p}^{4} \kappa \Gamma_{0}^{\frac{1}{2}}}\left(\frac{4(1-3 \kappa \bar{\lambda} \gamma)(2-k) n^{2} H_{0}^{3-2 k}}{3 \kappa \Gamma_{0}}\right. \\
& \left.\times\left(\frac{n+(m+2-n(3-2 k)) N}{n}\right)\right)^{\frac{3 n-m-4 n k-2}{2(m+2-n(3-2 k))}} .
\end{aligned}
$$

For high-dissipative regime, the inflationary observable turns out to be

$$
\begin{aligned}
n_{s}= & 1-\left(\frac{n-1}{6 n}+\frac{3-2 k}{6}+\frac{m}{12 n}+\frac{\Gamma_{0}}{3+\Gamma_{0}}\left(\frac{3 H_{0}}{\Gamma_{0}}+\frac{m}{6 n}\right)\right) \epsilon, \\
\alpha_{s}= & \left(\frac{9}{2}-\frac{(n-1)(n-2)}{6 n^{2}}-\frac{n-1}{2 n}-\frac{5}{3}(3-2 k)+\frac{3(2-2 k)(3-2 k)}{2 n}\right. \\
& +\frac{3 m(m-1)}{4 n}-\frac{m^{2}}{108 n}+\frac{m(n-1)}{108 n^{2}}-\frac{m}{12 n}+\frac{m(3-2 k)}{108 n}+\frac{r}{3+r}\left(\frac{H_{0}}{\Gamma_{0}}\right. \\
& \times \frac{2(n-1)}{9 n}-\frac{5 H_{0}(3-2 k)}{27 \Gamma_{0}}-\frac{m(3-2 k)}{27 n}+\frac{45 H_{0}}{12 \Gamma_{0}}+\frac{5 m}{12 n}-\frac{m^{2}}{108 n^{2}}
\end{aligned}
$$


Fig. $2 n_{s}-\alpha_{s}$ plane for low-dissipative regime (Left) and $n_{s}-\alpha_{s}$ plane for high-dissipative regime (Right) fixing $\bar{\lambda}=1$. Here, we fixed

$H_{0}=k=\kappa=1, \Gamma_{0}=0.5$ and $\gamma=0.16$. We choose specific values for $m=1,2,3$ and $n=2,3,4$, and number of e-folds $N=80$

Fig. 3 Left plot for $n_{s}-r$ plane during low-dissipative regime and right plot for $n_{s}-r$ plane during high-dissipative regime with contours of Plank 2018, for $\bar{\lambda}=0.5,1,1.5$. Here, we fixed $H_{0}=k=\kappa=1, \Gamma_{0}=0.5$, $m_{p}=1, \alpha=1$ and $\gamma=0.16$
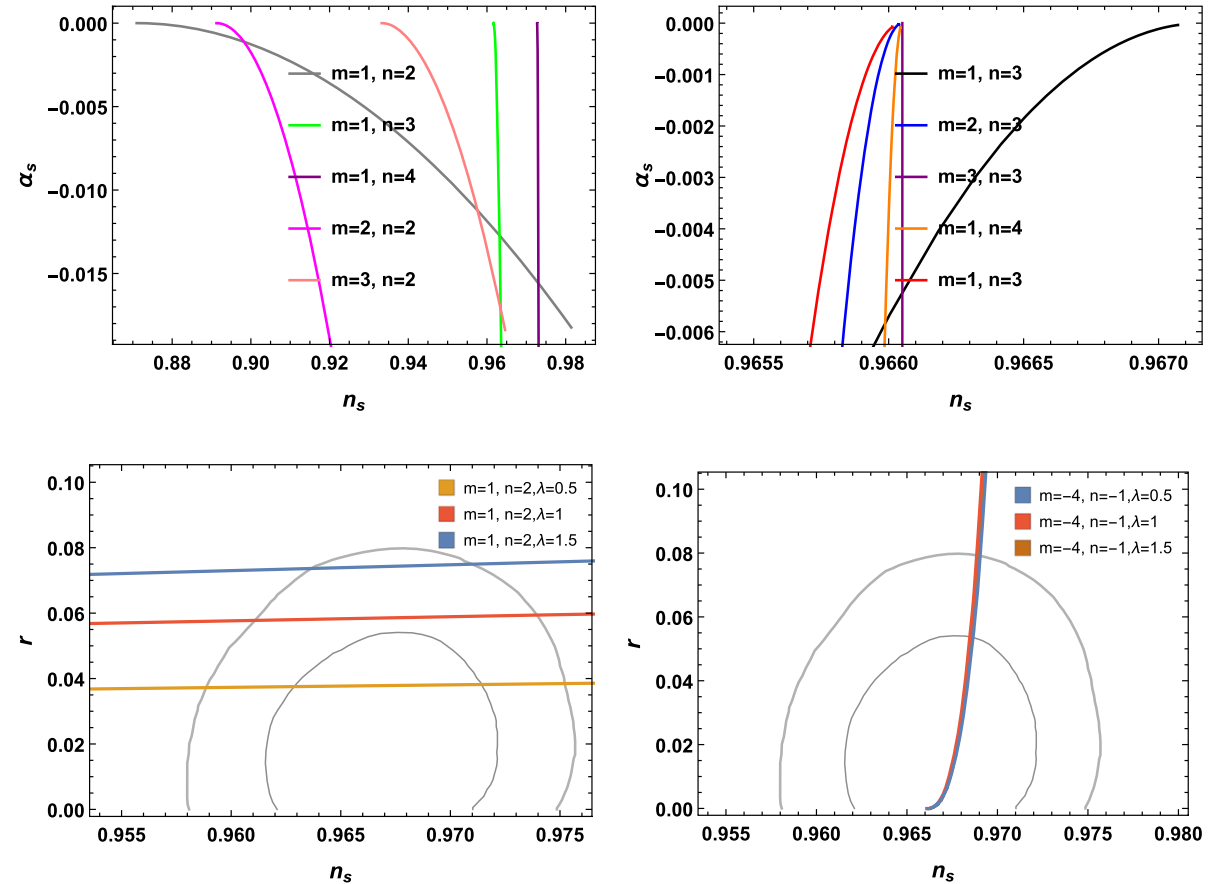

$$
\begin{aligned}
& \left.\left.+\frac{3 m(m-1)}{2 n}\right)+\frac{\Gamma_{0}^{2}}{\left(3+\Gamma_{0}\right)^{2}}\left(\frac{29 H_{0}^{2}}{18 \Gamma_{0}^{2}}+\frac{83 m H_{0}}{162 n \Gamma_{0}}+\frac{m^{2}}{27 n^{2}}\right)\right) \epsilon^{2}, \\
r= & \left(\frac{64(1-4 \kappa \bar{\lambda})}{9 m_{p}^{4} k}\right)\left(\frac{\alpha \pi^{4}}{4}\right)^{\frac{1}{4}} \Gamma_{0}^{-\frac{3}{4}} H_{0}^{\frac{13-8 k}{4}} n^{-\frac{3}{2}} \epsilon\left(\frac{(1-3 \kappa \bar{\lambda} \gamma)(2-k) n^{2} H_{0}^{3-2 k}}{3 \kappa \Gamma_{0}}\right. \\
& \left.\times\left(\frac{n+(m+2-n(3-2 k)) N}{n}\right)\right)^{\frac{13 n-3 m-8 n k}{4(m+2-n(3-2 k))}} .
\end{aligned}
$$

The above-mentioned details lay a theoretical framework of inflation. Furthermore, latest observational data from the Planck Satellite [60] provides further insight to the paradigm of WI. Also, it provides details about the most suitable data available on spectral index and its running. Figures 2 and 3 represent that the developed model with WI using Tsallis entropy is in good agreement with Planck 2018 data deep inside $2 \sigma$ level [3-5].

\section{Warm inflation using Barrow holographic dark energy within Rastall gravity}

Barrow [61] was motivated by the COVID-19 virus interpretation and revealed that quantum-gravitational effects might include complex fractal features on the structure of $\mathrm{BH}$. This complex formation escorts to finite volume besides infinite (or finite) area, and hence, a deformed $\mathrm{BH}$ entropy expression is

$$
S_{B}=\left(\frac{A}{A_{0}}\right)^{\Delta+1},
$$

where $A_{0}$ is the Planck area, $A$ is the standard horizon area, and the new term $\Delta$ lies in the interval $[0,1]$. It is worth mentioning the conventional quantum-corrected entropy with logarithmic corrections; though it simulates non-extensive Tsallis entropy, however, the physical principle and the intricate foundation are absolutely different. Thus, the purpose of this modified entropy relation just as the basis of HDE gives rise to BHDE [46], which is managed to improve phenomenology, and differentiating it from the standard framework of HDE [62-65]. The Barrow entropy expression is defined as [46]

$$
F(H)=\mathcal{C} \mathcal{L}^{-2(1-\Delta)},
$$

where $\mathcal{C}=3 c^{2} M_{p}^{2}, M_{p}$ is the Planck mass, $c^{2}$ is a dimensionless constant, and $\mathcal{L}$ represents the IR cutoff. Parameter $\mathcal{C}$ has dimension $[\mathcal{L}]^{-2(1-\Delta)}$. For vanishing $\Delta$, Eq. (40) will be the standard HDE, and it becomes constant under the limit $\Delta \rightarrow 1$. 
Fig. 4 Plot of $a(t)$ for $\bar{\lambda}=1,1.5$.

Here, we fixed

$H_{0}=1, \kappa=1, \Delta=0.04, \mathcal{C}=$

$10^{45}, \Gamma_{0}=0.5, \gamma=0.16$ and

$t_{0}=0.2$. We find the specific

values for $m=8,9,10$ and

$n=2,2.5,3$, which is valid for

this model

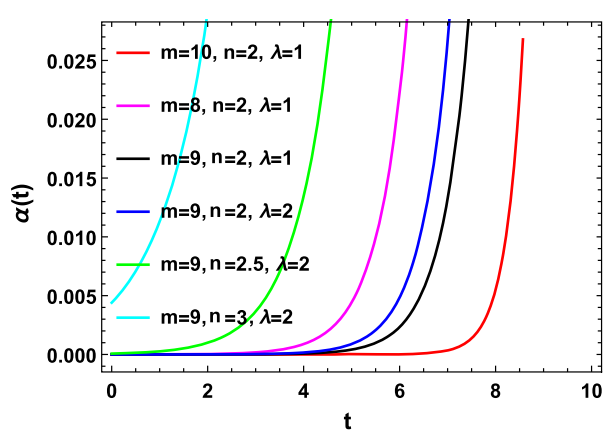

Considering the same power-law functions for $H$ and $\Gamma$, and setting $\epsilon=1$, we get the expression of inflaton at the horizon exit as

$$
B_{e}^{m-n(1-2 \Delta)+2}=\frac{4 \mathcal{C} n^{2}(1-3 \kappa \bar{\lambda} \gamma) H_{0}^{1-2 \Delta}(1-\Delta)}{3 \kappa \Gamma_{0}} .
$$

For this model, the number of e-folds turns out to be

$$
N=\int_{B}^{B_{e}}\left(\frac{3 \kappa}{4 \mathcal{C}(1-3 \kappa \bar{\lambda} \gamma)(1-\Delta)}\right) \frac{\Gamma_{0} B^{n} B^{m}}{n B^{n-1} H_{0}^{1-2 \Delta} B^{n(1-2 \Delta)}} d B,
$$

and this leads us to calculate inflaton field using BHDE entropy similar to Tsallis entropy case

$$
\begin{aligned}
B^{m-n(2-2 \Delta)+2}(t)= & \frac{(-m+n(2-2 \Delta)-2) B_{e}^{m-n(2-2 \Delta)+2} H_{0}\left(t-t_{0}\right)}{n} \\
& +B_{e}^{m-n(1-2 \Delta)+2}\left(1+\frac{(m-n(1-2 \Delta)+2) N}{n}\right)^{\frac{m-n(2-2 \Delta)+2}{m-n(1-2 \Delta)+2}} .
\end{aligned}
$$

The scale factor dependent upon BHDE entropy is attained in the following form

$$
a(t)=\exp \left(\frac{3 \kappa \Gamma_{0} B_{e}^{m-n(1-2 \Delta)+2}(-(m-n(1-2 \Delta)+2) N)}{4 \mathcal{C}(1-3 \kappa \bar{\lambda} \gamma)(1-\Delta) n H_{0}^{1-2 \Delta}(m-n(1-2 \Delta)+2)}\right) .
$$

Figure 4 represents the exponential nature of the scale factor, which is evaluated using BHDE entropy.

In this scenario, the parameter $\epsilon$ and the remaining parameters as a function of $\epsilon$ at horizon exit can be written as under

$$
\begin{aligned}
& \epsilon=\frac{n}{n+(m-n(1-2 \Delta)+2) N}, \quad \beta=\frac{m}{n} \epsilon, \quad \xi=\frac{H_{0}}{\Gamma_{0}} \epsilon, \quad v=\frac{(n-1)(n-2)}{n} \epsilon, \\
& \delta=\frac{m(m-1)}{n^{2}} \epsilon^{2}, \quad \psi=2 \Delta(1-2 \Delta) \epsilon, \quad \eta=\frac{n-1}{6 n} \epsilon, \quad \chi=(1-2 \Delta) \epsilon .
\end{aligned}
$$

The parameters $n_{s}, \alpha_{s}, r$ for low-dissipative regime are calculated as

$$
\begin{aligned}
n_{s}= & 1+\left(2-\frac{m}{2 n}+\frac{1}{2 n}+\frac{1}{3}\left(\frac{9 H_{0}}{\Gamma_{0}}+\frac{m}{n}\right)\right) \epsilon, \\
\alpha_{s}= & \left(-\frac{11(n-1)}{6 n}-\frac{5(1-2 \Delta)}{2}-\frac{m(m-1)}{2 n^{2}}+\frac{m^{2}}{2 n^{2}}-\frac{m(n-1)}{3 n^{2}}+\frac{m}{n}\right. \\
& -\frac{m(1-2 \Delta)}{n}+\frac{1}{3}\left(-\frac{9 H_{0}}{\Gamma_{0}}-\frac{m}{n}+\frac{3 m(n-1)}{2 n^{2}}+\frac{H_{0}(1-2 \Delta)}{\Gamma_{0}}\right. \\
& \left.\left.+\frac{m(1-2 \Delta)}{n}\right)+\frac{1}{9}\left(\frac{81 H_{0}^{2}}{\Gamma_{0}^{2}}+\frac{18 m H_{0}}{n \Gamma_{0}}+\frac{m^{2}}{n^{2}}\right)\right) \epsilon^{2}, \\
r= & \left(\frac{160 \mathcal{C}(4 \kappa \bar{\lambda}-1)}{39 m_{p}^{4} k}\right)\left(\frac{4}{\alpha \pi^{4}}\right)^{\frac{1}{4}} \Gamma_{0}^{\frac{3}{4}} H_{0}^{2(1-\Delta)-\frac{3}{4}} n^{\frac{3}{2}} \epsilon \frac{4 \mathcal{C}(1-3 \kappa \bar{\lambda} \gamma)(1-2 \Delta) n^{2} H_{0}^{1-2 \Delta}}{3 \kappa \Gamma_{0}} \\
& \left.\times\left(\frac{n+(m-n(1-2 \Delta)+2) N}{n}\right)\right)^{\frac{2 n(1-2 \Delta)-3 m / 4+3 n / 4-3(n-1) / 2}{m+2-n(1-2 \Delta)}} .
\end{aligned}
$$


Fig. 5 Left plot for $n_{s}-\alpha_{s}$ plane for BHDE entropy in low-dissipative regime and right plot for $n_{s}-\alpha_{s}$ plane for BHDE entropy in high-dissipative regime for $\bar{\lambda}=1$. Here, we fixed $H_{0}=1$, $\Delta=0.04, \mathcal{C}=10^{45}, \kappa=1$, $\Gamma_{0}=0.5$ and $\gamma=0.16$. We find the specific values for $m=7,8,9$ and $n=-1,-1.5,-2$ which is valid for this model by varying the number of e-folds

Fig. 6 Left plot for $n_{s}-r$ plane for BHDE entropy in

low-dissipative regime and right plot for $n_{s}-r$ plane for BHDE entropy in high-dissipative regime with contours of Plank 2018, for $\bar{\lambda}=0.5,1,1.5$. Here, we fixed $H_{0}=\kappa=1, \Gamma_{0}=0.5, m_{p}=1$, $\alpha=1, \Delta=0.04, \mathcal{C}=10^{45}$ and $\gamma=0.16$. We find the specific value for $m=-5$ and $n=7$, which is valid for this model by varying the number of e-folds
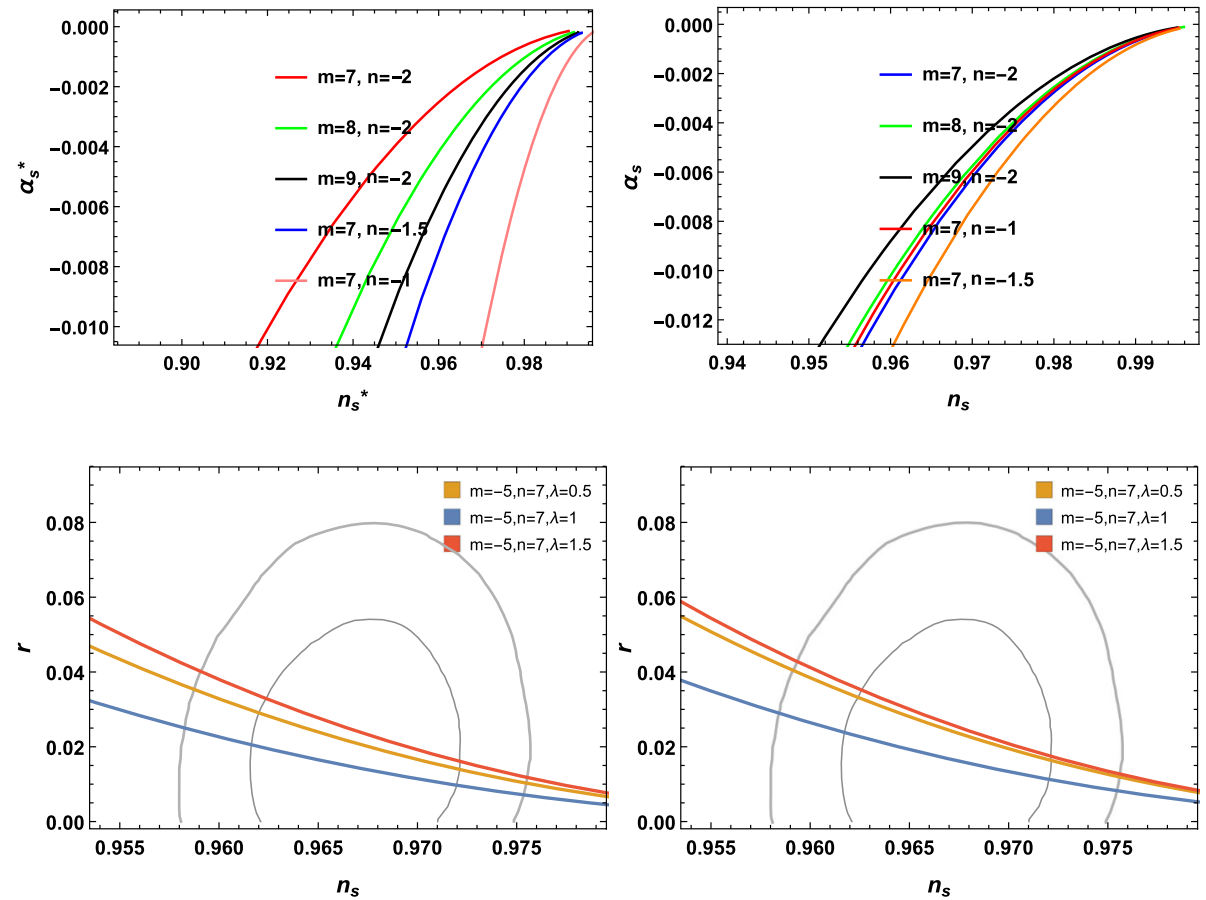

On the similar manner, $n_{s}, \alpha_{s}, r$ for high-dissipative regime are:

$$
\begin{aligned}
n_{s}= & 1+\left(\frac{9}{4}-\frac{(n-1)}{2 n}+\frac{3(1-2 \Delta)}{2}+\frac{3 m}{4 n}+\frac{\Gamma_{0}}{3+\Gamma_{0}}\left(\frac{21 H_{0}}{2 \Gamma_{0}}+\frac{3 m}{2 n}\right)\right) \epsilon, \\
\alpha_{s}= & \left(\frac{9}{2}-\frac{3(n-1)(n-2)}{2 n}-\frac{n-1}{n}-15(1-2 \Delta)+3 \Delta(2 \Delta-1)-\frac{3 m}{4 n^{2}}\right. \\
& +\frac{m(n-1)}{4 n^{2}}-\frac{3 m}{4 n}+\frac{3 m(1-2 \Delta)}{4 n}+\frac{\Gamma_{0}}{3+\Gamma_{0}}\left(\frac{6 H_{0}(n-1)}{n \Gamma_{0}}+\frac{15 m}{4 n}\right. \\
& \left.-\frac{15 H_{0}(1-2 \Delta)}{\Gamma_{0}}-\frac{3 m(1-2 \Delta)}{n}+\frac{135 H_{0}}{4 \Gamma_{0}}-\frac{3 m^{2}}{n^{2}}-\frac{27 m H_{0}}{4 n \Gamma_{0}}+\frac{3 m(m-1)}{2 n^{2}}\right) \\
& \left.+\frac{\Gamma_{0}^{2}}{\left(3+\Gamma_{0}\right)^{2}}\left(\frac{261 H_{0}^{2}}{2 \Gamma_{0}^{2}}+\frac{83 m H_{0}}{2 n \Gamma_{0}}+\frac{3 m^{2}}{n^{2}}\right)\right) \epsilon^{2}, \\
r= & \left(\frac{16 \mathcal{C}(4 \kappa \bar{\lambda}-1)}{39 m_{p}^{4} \kappa \Gamma_{0}^{-\frac{3}{4}}}\right)\left(\frac{4}{\alpha \pi^{4}}\right)^{\frac{1}{4}} H_{0}^{2(1-\Delta)-1 / 4} n^{\frac{3}{2}} \epsilon\left(\frac{4 \mathcal{C}(1-3 \kappa \bar{\lambda} \gamma)(1-2 \Delta) n^{2} H_{0}^{1-2 \Delta}}{3 \kappa \Gamma_{0}}\right. \\
& \left.\times\left(\frac{n+(m-n(1-2 \Delta)+2) N}{n}\right)\right)
\end{aligned}
$$

The left and right plots of Fig. 5 represent the compatibility of the $n_{s}-\alpha_{s}$ trajectories with Planck 2018 data as $\alpha_{s}=-0.0038$ for $n_{s}=0.967$. The $r-n_{s}$ trajectories shown in Fig. 6 are fitted well with recent observational data up to $2 \sigma$ level.

\section{Results and discussion}

In recent years, the idea of discussing inflation via DE models has become the center of attraction. Despite the extended research on the applications of holographic principle in late-time cosmology and DE epoch, there has not been any attempt in applying it at early universe, namely to obtain an inflationary realization of holographic origin. Such a consideration has equal footing with its well-studied late-time applications, and moreover, the decrease in the horizons at early times naturally increases holographic energy density at inflationary scales. In a paper [66], the holographic principle has been implemented at the early universe, and an inflation realization of the holographic origin is obtained. Confronting the theoretical predictions with Planck 2018 data, they showed that the agreement is perfect and in particular deep inside the $1 \sigma$ region. Darabi et al. [67] studied the inflation via logarithmic 
entropy-corrected HDE model with future event horizon, particle horizon and Hubble horizon cutoffs and compared their results with those of obtained in the study of inflation by HDE model [68].

Recently, an author in Ref. [56] modified the standard Friedmann equations using Tsallis entropy to discuss WI. This approach is quite different, and the solutions are easy to handle and well presented than already existed intermediate/logamediate technique. Motivating by this idea, we try to extend the basic scenario to more subtle constructions. In the present work, we are interested in investigating Barrow holographic and Tsallis inflation, to acquire a successful inflation triggered by their energy densities. As we show, although the basic scenario is very simple and natural, it can be very efficient and results in inflationary observables in perfect agreement with observations. We considered BHDE entropy along with Tsallis entropy to modify the Friedmann equations and continuity equation with general function of Hubble parameter within RG framework. We evaluated the exact solutions of the inflaton field, scalar potential, Hubble parameter, scale factor and the dissipation coefficient under slow-roll approximation. For both models, we evaluated inflationary observable including slow-roll parameters, e-folding number, scalar/tensor power spectra, scalar spectral index, running of scalar spectral index and the tensor-to-scalar ratio. The work has been done for both high- and low-dissipative regimes.

Figures 1 and 4 represent that the evaluated forms of the scale factors in Eqs. (36) and (41) for Tsallis and BHDE entropies, respectively, are positive and monotonically increasing. The behavior of the factor $a(t)$ is predicted for different values of free parameters $m, n, \bar{\lambda}, \Delta, \delta, \Gamma_{0}$. For both models, $n_{s}-\alpha_{s}$ trajectories are plotted in Figs. 2 and 5 , respectively. The left graphs are plotted for low-dissipative regimes, while right one for high dissipation. Both of the figures show good compatibility of the parameter, running of spectral index with Planck 2018 probe for constraint values of the involved model parameters. For both low- and highdissipative regimes, it is noticed that $\alpha_{s}-n_{s}$ trajectories are less sensitive for Rastall parameter $\bar{\lambda}$, while more sensitive for the variation of free parameters $m, n$. The tensor-to-scalar ratio is plotted versus $n_{s}$ in Fig. 3 for Tsallis entropy and in Fig. 6 for BHDE entropy. The left and right plots of both of the figures clearly depicted that the developed model during low/high-dissipative regimes is in good agreement ( $2 \sigma$ level) with Planck data.

In a nutshell, we have observed that this technique is more useful than existed techniques as we are able to evaluate exact solution of the inflationary model, which are simplified and easy to handle. The results are quite interesting and physically viable. It is worth mentioning here that results for Tsallis entropy are reduced to [56] for vanishing addition Rastall parameter $\bar{\lambda}$, while BHDE model with inflation is a new idea and not implemented in GR yet.

Data Availability Statement All data generated or analyzed during this study are included in this published article (and its supplementary information files).

\section{References}

1. A.H. Guth, Phys. Rev. D 23, 347 (1981)

2. G. Hinshaw et al., Astrophys. J. Suppl. 208, 19 (2013)

3. N. Aghanim et al., Planck 2018 results. VI. Cosmological parameters. Astron. Astrophys. 641, A6 (2020)

4. Y. Akrami et al., Planck 2018 results. X. Constraints on inflation. Astron. Astrophys. 641, A10 (2020)

5. Y. Akrami et al., Planck 2018 results. VII. Isotropy and statistics of the CMB. Astron. Astrophys. 641, A7 (2020)

6. J. Martin, C. Ringeval, V. Vennin, Phys. Dark Universe 75, 5 (2014)

7. T. Buchert, A.A. Coley, H. Kleinert, B.F. Roukema, D.L. Wiltshire, Int. J. Mod. Phys. D 25, 1630007 (2016)

8. K. Sato, Mon. Not. R. Astron. Soc. 195, 467 (1981)

9. A. Linde, Phys. Lett. B 108, 389 (1982)

10. A. Albrecht, P. Steinhardt, Phys. Rev. Lett. 48, 1220 (1982)

11. A. Linde, Phys. Lett. B 129, 177 (1983)

12. A. Berera, Phys. Rev. Lett. 75, 3218 (1995)

13. P. Rastall, Phys. Rev. D 6, 3357 (1972)

14. P. Rastall, Can. J. Phys. 54, 66 (1976)

15. L.L. Smalley, Nuovo Cim. B 80, 42 (1984)

16. T.S. Almeida, M.L. Pucheu, C. Romero, J.B. Formiga, Phys. Rev. D 89, 064047 (2014)

17. C.E.M. Batista, J.C. Fabris, O.F. Piattella, A.M. VelasquezToribio, Eur. Phys. J. C 73, 2425 (2013)

18. J.C. Fabris et al., Int. J. Mod. Phys. Conf. Ser. 18, 67 (2012)

19. C.E.M. Batista et al., Phys. Rev. D 85, 084008 (2012)

20. G.W. Gibbons, S.W. Hawking, Phys. Rev. D 15, 2738 (1977)

21. L. Parker, Phys. Rev. D 3, 346 (1971). [Erratum. Phys. Rev. D 3(1971)2546]

22. L.H. Ford, Phys. Rev. D 35, 2955 (1987)

23. S.H. Pereira, C.H.G. Bessa, J.A.S. Lima, Phys. Lett. B 690, 103 (2010)

24. S. Perlmutter et al., Astrophys. J. 517, 565 (1999)

25. A.G. Riess et al., Astron. J. 116, 1009 (1998)

26. L. Amendola, Phys. Rev. D 62, 043511 (2000)

27. E. Karimkhani, A.K. Mohammadi, Astrophys. Space Sci. 364, 177 (2019)

28. F. Arevalo, P. Cifuentes, S. Lepe, F. Pena, Astrophys. Space Sci. 2, 899 (2014)

29. R. Saleem, M.J. Imtaiz, Class. Quantum Gravity 37, 065018 (2020)

30. S.D. Campo, J.C. Fabris, R. Herrera, W. Zimdhal, Phys. Rev. D 83, 123006 (2011)

31. C. Tsallis, J. Stat. Phys. 52, 479 (1988) 
32. M.L. Lyra, C. Tsallis, Phys. Rev. Lett. 80, 53 (1998)

33. G. Wilk, Z. Wlodarczyk, Phys. Rev. Lett. 84, 2770 (2000)

34. C. Rovelli, Phys. Rev. Lett. 77, 3288 (1996)

35. A. Ashtekar, J. Baez, A. Corichi, K. Krasnov, Phys. Rev. Lett. 80, 904 (1998)

36. S.D. Odintsov, Int. J. Mod. Phys. A 16, 3273 (2001)

37. J. Zhang, Phys. Lett. B 668, 353 (2008)

38. Y.F. Cai, J. Liu, H. Li, Phys. Lett. B 690, 213 (2010)

39. S. Das, S. Shankaranarayanan, S. Sur, Phys. Rev. D 77, 064013 (2008)

40. N. Radicella, D. Pavon, Phys. Lett. B 691, 121 (2010)

41. S. Nojiri, S.D. Odintsov, E.N. Saridakis, Eur. Phys. J. C 79, 242 (2019)

42. Q. Huang, H. Huang, J. Chen, L. Zhang, F. Tu, Class. Quantum Gravity 36, 175001 (2019)

43. S. Ghaffari et al., Eur. Phys. J. C 78, 706 (2018)

44. E.N. Saridakis, K. Bamba, R. Myrzakulov, F.K. Anagnostopoulos, JCAP 18, 012 (2018)

45. S. Ghaffari, H. Moradpour, V.B. Bezerra, J. Morais Graaa, I. Lobo, Phys. Dark Universe 23, 100246 (2019)

46. E.N. Saridakis, Phys. Rev. D 102, 123525 (2020)

47. G. Chakraborty et al., Symmetry 13, 562 (2021)

48. P. Adhikary, S. Das, S. Basilakos, E.N. Saridakis, Phys. Rev. D 104, 123519 (2021)

49. F.K. Anagnostopoulos, S. Basilakos, E.N. Saridakis, Eur. Phys. J. C 80, 1-9 (2020)

50. A.A. Mamon, A. Paliathanasis, S. Saha, Eur. Phys. J. Plus 136, 1-14 (2021)

51. A. Sarkar, S. Chattopadhyay, Int. J. Geom. Methods Mod. Phys. 18, 2150148 (2021)

52. U. Debnath et al., Eur. Phys. J. C 72, 1-6 (2012)

53. S.I. Nojiri, S.D. Odintsov, V. Faraoni, Phys. Rev. D 104, 084030 (2021)

54. S.I. Nojiri, S.D. Odintsov, V. Faraoni, arXiv preprint arXiv:2201.02424

55. G. Chakraborty, S. Chattopadhyay, Zeitschrift für Naturforschung A 76, $43-64$ (2021)

56. O. Trivedi, arXiv:2008.05899

57. A. Taylor, A. Berera, Phys. Rev. D 62, 083517 (2000)

58. A.R. Liddle, D.H. Lyth, Phys. Rep. 231, 1 (1993)

59. Y. Akrami, et al., arXiv:1807.06211

60. N. Aghanim, et al., arXiv:1807.06209

61. J.D. Barrow, Phys. Lett. B 808, 135643 (2020)

62. M.P. Dabrowski, V. Salzano, Phys. Rev. D 102, 064047 (2020)

63. E.M.C. Abreu, J.A. Neto, Phys. Lett. B 810, 135805 (2020)

64. J.D. Barrow, S. Basilakos, E.N. Saridakis, Phys. Lett. B 815, 136134 (2021)

65. U.K. Sharma, G. Varshney, V.C. Dubey, Int. J. Mod. Phys. D 30, 2150021 (2021)

66. S. Nojiri, S.D. Odintsov, E.N. Saridakis, Phys. Lett. B 797, 134829 (2019)

67. F. Darabi, F. Felegary, M.R. Setare, Eur. Phys. J. C 76, 703 (2016)

68. B. Chen, M. Li, Y. Wang, Nucl. Phys. B 774, 256 (2007) 